Please quote as: Billert, M. \& Peters, C. (2019). Die Digitalisierungsstraße für die Stadt der Zukunft. Innovative Verwaltung, 41, 172-189. doi: 10.1365/s40702-01800490-6 


\title{
Die Digitalisierungsstraße für die Stadt der Zukunft - Kollaborative Entwicklung eines Portals für bürger-initiierte Dienstleistungsentwicklung im Kontext einer Smart City
}

\author{
Matthias Simon Billert (iD • Christoph Peters
}

Eingegangen: 31. August 2018 / Angenommen: 21. Dezember 2018 / Online publiziert: 17. Januar 2019 (C) Springer Fachmedien Wiesbaden GmbH, ein Teil von Springer Nature 2019

Zusammenfassung Immer mehr Menschen mit unterschiedlichen Kompetenzen leben in Städten. Dabei stehen Stadtverwaltungen vor der großen Herausforderung die Kompetenzen der BürgerInnen nicht außen vor zu lassen, sondern sinnvoll einzubinden, um mit neuen Dienstleistungen einen vielseitigen, nachhaltigen und lebenswerten Raum für ihre BürgerInnen zu schaffen. Hierfür sollten BürgerInnen aktiv in den Entwicklungsprozess eingebunden werden, denn sie sind Experten ihres Alltags. In den meisten Fällen ist die entsprechende Bürgerbeteiligung jedoch nicht gegeben und eine nötige kollaborative Entwicklung von neuen, bürgerzentrierten Dienstleistungen bleibt aus. Um dem entgegenzuwirken kann durch die Verwendung von Informations- und Kommunikationstechnologien ein Lösungsansatz geschaffen werden, der die kollaborative Dienstleistungsentwicklung von BürgerInnen und der Stadtverwaltung realisiert. Unter Verwendung von Design Science Research und Collaboration Engineering bauen wir in mehreren, kollaborativen Zyklen die „Digitalisierungsstraße“, ein Portal, das die BürgerInnen systematisch durch die Entwicklung eigener Dienstleistungsideen führt. Dabei haben die BürgerInnen die Möglichkeit auf Basis eines multimedialen, medienbruchfreien Ansatzes, (1) ihren Projektvorschlag zu beschreiben, (2) ihren Projektvorschlag mittels einer webbasierten Zeichenumgebung visuell darzustellen, (3) ihren Projektvorschlag als „Elevator Pitch“ videobasiert zu präsentieren. Basierend auf der Literatur und mehreren Workshops sowie auf Basis von Anforderungen zur kollaborativen Dienstleistungserstellung tragen wir zum vorhandenen Wissen der kollaborativen Portalentwicklung bei, indem wir bürger-

\author{
M. S. Billert $(\bowtie) \cdot$ C. Peters \\ University of Kassel, Pfannkuchstraße 1, 34121 Kassel, Deutschland \\ E-Mail: matthias.billert@uni-kassel.de \\ C. Peters \\ E-Mail: christoph.peters@uni-kassel.de \\ C. Peters \\ University of St. Gallen, Müller-Friedberg-Straße 8, 9000 St. Gallen, Deutschland
}


initiierte Dienstleistungsideen auf Basis eines bürgerzentrierten Ansatzes entwickelt haben. Durch die kollaborative Entwicklung der Digitalisierungsstraße wird außerdem ersichtlich wie eine eigenständige Portallösung entwickelt wurde, um die Bürgerbeteiligung von einer einfachen Idee zu einer durchdachten Dienstleistungslösung unter Verwendung von multimedialen Elementen zu steigern.

Schlüsselwörter Smart City · Bürgerbeteiligung · Gestaltungsorientierter Forschungsansatz $\cdot$ Kollaborative Entwicklung · Dienstleistungssystementwicklung

\title{
The Digitalization Street for the City of the Future - Collaborative Development of a Portal for the Development of Citizen-Initiated Services in the Context of a Smart City
}

\begin{abstract}
More and more people with different skills live in cities. City administrations are faced with the great challenge not to ignore the competences of citizens, but to integrate them in order to create with new services a versatile, sustainable, and livable area for their citizens. To this end, citizens should be actively involved in the development process because they are experts of their everyday lives. However, in most cases, there is no corresponding citizen participation and the necessary collaborative development of new, citizen-centered services is not taking place. To counteract this, the use of information and communication technologies can create a solution approach that realizes the collaborative service development of citizens and the city administration. Using design science research and collaboration engineering, we are building in several collaborative cycles the "digitalization street", which is a portal that systematically guides citizens through the development of their own service ideas. On the basis of a multimedia, media-break-free approach, citizens have the possibility to (1) describe their project proposal, (2) visualize their project proposal using a web-based drawing environment, (3) present their project proposal as an elevator pitch on video. Based on the literature and several workshops as well as on requirements for collaborative service engineering, we contribute to the existing knowledge of collaborative portal development by developing citizeninitiated service ideas based on a citizen-centered approach. The collaborative development of the digitalization street also shows how an independent portal solution was developed to increase citizen participation from a simple idea to a well-thoughtout service solution using multimedia elements.
\end{abstract}

Keywords Smart city · Citizen participation · Design science research · Collaboration engineering $\cdot$ Service system engineering

\section{Einleitung}

Immer mehr Menschen mit unterschiedlichen Kompetenzen leben in Städten (United Nations, Department of Economic and Social Affairs, Population Division 2017). Dies führt dazu, dass Stadtverwaltungen vor einer immer größeren Herausforderung stehen, die Kompetenzen der BürgerInnen nicht außen vor zu lassen, sondern 
einzubinden und bürger-zentrierte Dienstleistungen zu erstellen (Letaifa 2015). Um den neuen Herausforderungen entgegenzuwirken, zielen Städte darauf ab, sich zu intelligenten Städten, sog. Smart Cities, zu wandeln, indem sie durch die neuen Dienstleitungen einen vielseitigen, nachhaltigen und lebenswerten Raum für die BürgerInnen schaffen (Castelnovo 2016). Dabei wird der Markt für neue Geschäftsmöglichkeiten und potentielle Dienstleistungen im Kontext einer Smart City in 2020 auf 1,565 Billionen Dollar geschätzt (Castelnovo 2016).

Bisher lag der Fokus einer Smart City auf dem kontinuierlichen, ortsunabhängigen Zugang zu Informationen mit Datenerfassung und ständiger Kontrolle sowohl mittels Sensoren der Informations- und Kommunikationstechnologie (IKT) als auch durch die Datenspeicherung und -verarbeitung (Cosgrave et al. 2013). Jedoch reicht die Verwendung von IKT alleine nicht aus, um eine Stadt in eine Smart City zu wandeln und die Lebensqualität der Stadtbewohner zu steigern (van Waart et al. 2016). Deshalb ist bei Smart Cities auch eine Veränderung zu verzeichnen: von einer technologisch, fokussierten Infrastruktur hin zu einer aktiven Einbindung von Bürgern durch die Verwendung von intelligenter Technologie (Paskaleva et al. 2018). BürgerInnen werden dabei als aktive Teilnehmer und Experten ihres alltäglichen Umfelds in die Entwicklung und Gestaltung von Dienstleistungen eingebunden, wodurch neue Formen der Bürgerbeteiligung gefördert werden.

Die Praxis sieht jedoch anders aus und aktuell werden BürgerInnen in den meisten Fällen nicht in die Dienstleistungsentwicklung einbezogen. In den wenigen Ausnahmefällen endet die Beteiligung jedoch in der Ideenphasen (Letaifa 2015), während die Entwicklungs- und Implementierungsphase von Dienstleistungen nicht weiter verfolgt wird. Deshalb suchen Stadtverwaltungen nach einer neuen Möglichkeit, BürgerInnen aktiv in den Erstellungsprozess zu involvieren. Allerding scheitern vorhandenen Ansätze der Stadtverwaltung daran, dass die BürgerInnen die Ansätze nicht akzeptieren (van Waart et al. 2016). Wohingegen Bürgerinitiativen um die Aufmerksamkeit und Unterstützung der Stadtverwaltung kämpfen müssen. Die Herausforderung der Städte, das enorme Potential neuer Dienstleistungen und die aktive Einbindung der BürgerInnen als Experten ihres Alltages haben dazu geführt, dass wir auf Basis eines bürger-zentrierten Ansatzes die Digitalisierungsstraße entwickelt haben. Dabei werden BürgerInnen über ein Portal systematisch im Dienstleistungserstellungsprozess begleitet - von der Idee bis zur Lösungspräsentation.

Damit bürger-initiierte Dienstleistungen umgesetzt werden können, müssen beide Akteure, Stadt und BürgerInnen, kollaborativ zusammenarbeiten und in den Erstellungsprozess des Portals involviert werden. Daraus ergibt sich die Frage: Wie muss der kollaborative Erstellungsprozess des Portals durchgeführt werden, damit bürgerinitiierte Dienstleistungen im Kontext einer Smart City erstellt werden können? Um die Fragen zu beantworten, geben wir in diesem Beitrag zunächst einen Einblick in die Literatur der Bürgerbeteiligung sowie der kollaborativen Dienstleistungserstellung. Außerdem zeigen wir auf, wie die Digitalisierungsstraße mit ihren drei Schritten konzeptioniert, kollaborativ entwickelt und in den einzelnen Iterationen evaluiert wurde. Im Anschluss an die Zusammenfassung unserer Ergebnisse geben wir einen Ausblick auf theoretische und praktische Beiträge. Abschließend zeigen wir zukünftige Forschungsmöglichkeiten und Weiterentwicklungen des Portals einschließlich seiner Limitation auf. 


\section{Von der Bürgerbeteiligung zur kollaborativen Dienstleistungserstellung}

\subsection{Bürgerbeteiligung in einer Smart City}

Es existieren bereits viele verschiedene Möglichkeiten sich als BürgerInnen zu beteiligen. Dabei fängt Beteiligung schon damit an, dass BürgerInnen sich auf der städtischen Webseite über das politische Geschehen informieren oder per Onlineformular Anregungen und Feedback an die Stadtverwaltung übermitteln. Das Ziel der Bürgerbeteiligung liegt in der lebenswerten und intelligenten Gestaltung der städtischen Gebiete hin zu einer Smart City (Mueller et al. 2018).

Um das Ziel einer verbesserten Bürgerbeteiligung zu erreichen, müssen die BürgerInnen in die Entwicklung von bürger-zentrierten Dienstleistungen einbezogen werden. Die Einbeziehung von Bürgern durch den Einsatz von IKT mit dem Ziel der Stadtverwaltung einen höheren kollaborativen, integrativen und partizipativen Charakter für instrumentale und intrinsische Zwecke zu verleihen, wird als E-Partizipation bezeichnet (Naranjo Zolotov et al. 2018). Damit wird aus der E-Partizipation ein strategisches Instrument der Stadtverwaltung zur Förderung der Gesellschaft und Steigerung der Bürgerbeteiligung. Die Bürgerbeteiligung in Kombination mit dem Einsatz von neuen Technologien wird als ein neuer Trend gesehen. Um die Nachhaltigkeit von Städten gewährleisten zu können, müssen gewillte BürgerInnen unter Verwendung von IKT an der Optimierung von städtischen Prozessen teilnehmen (Gabrys 2014). Dadurch werden BürgerInnen zu Sensoren, die rechnergestützte Technologien verwenden, um Feedback zu geben. Dies wird auch Citizen Sensing genannt. Citizen Sensing bietet dabei eine Grundlage für eine entwicklungsorientierte und kreative Beteiligung unter Einsatz von medialen und partizipativen Technologien, um bürger-initiierte Dienstleistungen umzusetzen. Für eine erfolgreiche Entwicklung der Bürgerbeteiligung hat Frecks (2015) drei Faktoren identifiziert, die erfüllt sein müssen: Einerseits muss die Existenz einer Terminologie, die auf die Politik angewendet wird, vorhanden sein. Eine weitere Voraussetzung ist die Gewährleistung der Partizipationskontrolle für die BürgerInnen während der Umsetzungsarbeit. Schlussendlich muss ein gemeinsames Verständnis der einzelnen Beteiligungsrollen im Kooperationsprozess durch Kategorien definiert werden.

Der Einsatz von IKT-Ansätzen verstärkt in diesem Fall die Beziehung zu Bürgern, was wiederum dazu führt, dass neue Kommunikationsansätze gefördert werden müssen, um einen Dialog zu den Bürgern zu eröffnen (Viale Pereira et al. 2017). Hierfür hat die internationale Vereinigung IAP2 Föderation ${ }^{1}$ ein Beteiligungsspektrum für die Förderung und Verbesserung der Öffentlichkeitsbeteiligung entwickelt. Das öffentliche Beteiligungsspektrum setzt sich aus fünf Ebenen zusammen und zeigt den steigenden Einfluss auf die Entscheidung durch eine Beteiligung der Öffentlichkeit: Die erste Ebene „Informieren“ beschäftigt sich damit, die Öffentlichkeit mit ausgewogenen und objektiven Informationen zu versorgen, um sie beim Verständnis des Problems, der Alternativen, Möglichkeiten und/oder Lösungen zu unterstützen. In der zweiten Ebene „Konsultieren“ geht es darum, ein öffentliches Feedback über

\footnotetext{
1 https://www.iap2.org/.
} 
Analysen, Alternativen und/oder Entscheidungen zu erhalten. Auf der dritten Ebene des „Involvieren“ wird während des gesamten Prozesses direkt mit der Öffentlichkeit zusammengearbeitet, um sicherzustellen, dass die Anliegen und Bestrebungen der Öffentlichkeit konsequent verstanden und berücksichtigt werden. Die vierte Ebene „Kollaborieren“ fokussiert die Partnerschaft mit der Öffentlichkeit in jedem Aspekt der Entscheidung, einschließlich der Entwicklung von Alternativen und der Identifizierung der bevorzugten Lösungen. In der letzten und fünften Ebene „Empowern“ geht es um die endgültige Entscheidungsfindung, die in die Hände der Öffentlichkeit gelegt wird. In unserem Fall spielt die Ebene der Kollaboration des IAP2 Spektrums die größte Rolle, da die BürgerInnen als Experten ihres Alltags in die politische Entscheidungsfindung und die Entwicklung von Dienstleistungsideen integriert werden. Um die bürger-initiierte Dienstleistungsentwicklung gewährleisten zu können, wird das Portal in einem kollaborativen Prozess entwickelt.

\subsection{Kollaborative Dienstleistungserstellung im Dienstleistungssystem Stadt}

Dienstleistungen zeichnen sich dadurch aus, dass der Nutzen durch die Zusammenarbeit von Kunden und Anbieter entsteht. Mehrere Akteure tragen durch ihre Zusammenarbeit (Value Cocreation) in einem bestimmten Kontext zur Wertschöpfung der Dienstleistung bei (Böhmann et al. 2014). Die Prozesse, Leistungen oder Taten für einen selbst oder für andere beruhen dabei auf der Anwendung von Fähigkeiten und Wissen (Kompetenzen). Die Gestaltung, Entwicklung und Umsetzung von Dienstleistungen unter der Verwendung von geeigneten Methoden, Werkzeugen und Vorgehensmodellen nennt man Service Engineering (Leimeister 2012). Beim Service Engineering gilt es, sich die Potentiale der Innovationskraft, Qualität, Wirtschaftlichkeit und vor allem der Kundenorientierung zu Nutze zu machen (Leimeister 2012). Die gemeinsame Wertschöpfung (joint value creation) von Dienstleistungen durch verschiedene Akteure geschieht dabei in einem Dienstleistungssystem, das als grundlegende Entität mittels Handlungen auf das Erreichen eines gegenseitigen Nutzens abzielt (Leimeister 2012).

Durch das große und vielfältige Angebot an städtischen Dienstleistungen wird die Stadt zu einem komplexen, sozio-technischen System, das bestehende Dienstleistungen verbessern und neue Dienstleistungen erschließen muss, um die Lebensqualität der BürgerInnen zu verbessern (Gutierrez et al. 2018). Komplexe, sozio-technische Systeme die eine interaktive und gemeinsame Wertschöpfung ermöglichen sind sogenannte Dienstleitungssysteme (Böhmann et al. 2014). In einem Dienstleistungssystem trägt das Zusammenspiel von Technologien, Informationen, Organisationen und Menschen zu einem gegenseitigen Nutzen bei. So werden im Prozess der gemeinsamen Wertschöpfung BürgerInnen als zentrale Akteure in die Konzeptionierung und Umsetzung von Dienstleistungen mitinvolviert, um zu einer soziologische, wirtschaftliche und technologische Entwicklung der Stadt beizutragen (Gutierrez et al. 2018). Für die Ermittlung der Anforderungen zur Erstellung von Dienstleistungen müssen die Leistungen und Ressourcen des komplexen Dienstleistungssystems Stadt analysiert werden. Auf unseren Kontext der Digitalisierungsstraße bezogen, beinhaltet die Technologiekomponente alle technischen Komponenten der Plattform, auf der das Portal zur Sammlung und Generierung von Dienstleistungsideen ange- 
boten wird. Zur Informationskomponente zählen jegliche für die BürgerInnen und die Stadtverwaltung relevante Informationen die nutzer-, nutzungs- und nutzenorientiert sind sowie alle relevanten Richtlinien und Gesetze der Stadtverwaltung. Bei der Organisationskomponente spielen andere interne und externe Dienstleistungssysteme sowie Unternehmen eine Rolle. Die Mitarbeiter der Stadtverwaltung und die BürgerInnen, die beide jeweils als Prosument des Dienstleistungssystems Stadt agieren, sind der Komponente Mensch zuzuordnen. Dabei ist das Zusammenspiel von Bürgern und der Stadtverwaltung von essentieller Bedeutung, damit der Bürger als Individuum sein Wissen und seine Fähigkeiten zur Erstellung von Dienstleistungsideen einbringen kann.

\section{Methodische Ansätze in der kollaborativen Designforschung}

\subsection{Design Science Research}

Zur Gestaltung eines Portals für die Erstellung von bürger-initiierten Dienstleistungen müssen einerseits die praktischen, relevanten Problemstellungen und andererseits die bisher gesicherten, wissenschaftlichen Erkenntnisse aus der Wissensbasis einfließen. Um dies systematisch zu tun, folgen wir dem Forschungsansatz „The Three Cycle of Design Science Research“ von Hevner (2007) verwendet. Dabei handelt es sich um einen designorientierten Forschungsansatz, bei dem die drei Zyklen „Rigor Cycle“, „Relevance Cycle“, und „Design Cycle“ bei jeder Iteration durchlaufen werden.

Der Rigor Cycle verbindet die vorhandene Wissensbasis mit den Design-Aktivitäten, indem fundierte Methoden und Theorien sowie Expertisen und Erfahrungen aus der Wissensbasis übertragen werden und in die Erstellung des geplanten DesignArtefakts einfließen (Hevner 2007). Die Erkenntnisse aus der Entwicklung und Forschung fließen anschließend wieder in die Wissensbasis und erweitern diese kontinuierlich (Gregor und Hevner 2013). Eine Voraussetzung ist, dass das zu erstellende Design auf Forschungsbeiträgen basieren muss, um die Innovation gewährleisten zu können (Hevner 2007). Die Anforderungen des kontextuellen Praxisumfelds des Forschungsprojekts werden mit den Forschungsartefakten der Wissenschaft und den Design-Aktivitäten über den Relevance Cycle verbunden (Hevner 2007). Dadurch wird die Definition von Akzeptanzkriterien für eine Bewertung der Ergebnisse und die Identifikation von Chancen sowie Problemen aus dem realen Anwendungsumfeld vorausgesetzt (Hevner 2007). Durch das Zusammenspiel der Personen im Anwendungsbereich und den technischen Systemen sowie Organisationssystemen werden die gemeinsamen Ziele erreicht. In einer engen Iteration der Forschungsaktivitäten wird im zentralen Design Cycle die Erstellung und Bewertung des zu entwerfenden Artefakts als auch der Designprozesse fokussiert (Hevner 2007). Auf Basis von Anforderungen erfolgt eine Bewertung der Alternativen und das anschließende Feedback dient der Verfeinerung des Designs. Der Design Cycle ist von den beiden anderen Zyklen abhängig und nutzt deren Input. Bei der Erstellung und Bewertung des Artefakts fungiert der Design Cylce dann allerdings unabhängig und wird solange durchlaufen bis der Entwurf als zufriedenstellend deklariert werden kann (Hevner 


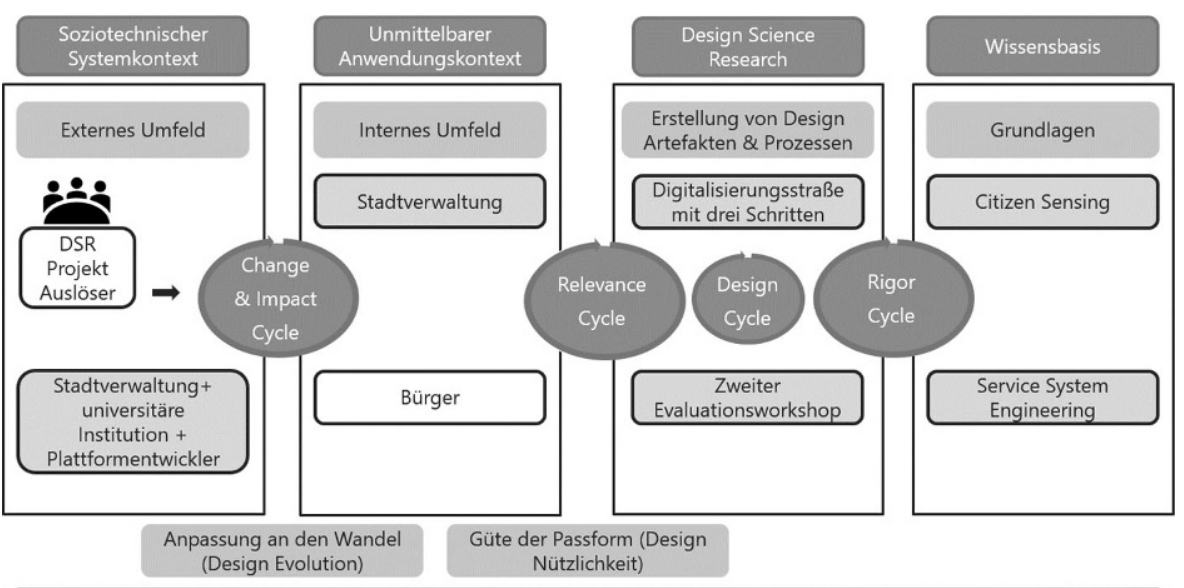

Framework for Evaluation in Design Science Research

Abb. 1 Eigene Darstellung der zweiten Iteration in Anlehnung an Drechsler und Hevner (2016)

2007). Voraussetzung für ein erfolgreiches Durchlaufen ist eine nachvollziehbare Argumentation für die Erstellung des Artefakts sowie eine fundierte Bewertung. Hierfür bietet es sich an, zunächst mit Labortests und Experimenten das Artefakt ausgiebig zu testen bevor Feldversuche mit Nutzern durchgeführt werden und neue Erkenntnisse über die beiden Zyklen zurückgespielt werden, um die bestehende Wissensbasis und das Umfeld zu erweitern. Damit die Korrektheit und Genauigkeit des Artefakts gewährleistet werden kann, muss das Artefakt kontinuierlich evaluiert werden. Für eine Evaluierung des Artefakts dient das „Framework for evaluation in design science (FEDS) Research“ von Venable et al. (2016). Dabei werden zunächst die Evaluationsziele festgelegt, die Bewertungsstrategie ausgewählt, die Eigenschaften der Auswertung definiert und die einzelnen Auswertungsepisoden entworfen.

Um einen Rahmen für die Entwicklung von bürgerinitiierten Dienstleistungen zu schaffen, wird für die Erstellung der Digitalisierungsstraße in einer ersten Iteration einerseits auf die Wissensbasis zum Thema Bürgerbeteiligung zurückgegriffen. Andererseits werden Methoden und Instrumente zur Erstellung und Analyse von Dienstleistungen sowie Dienstleistungssystemen über den Rigor Cycle bezogen. Auf der Seite des Umfelds wird über den Relevance Cycle ein mit Experten durchgeführte Anforderungsworkshop sowie das Wissens aus dem kontinuierlichen Austausch mit den einzelnen Stakeholdern zur Bedarfserhebung übertragen. Auf Basis dessen wird eine erste Iteration der Digitalisierungsstraße mit dem Design Cycle erstellt und anschließend in einem Workshop evaluiert. Die Erkenntnisse aus dem Workshop erweitern die Wissensbasis zum Thema Bürgerbeteiligung und Dienstleistungserstellung sowie die Bedürfnisse der Stakeholder bezogen auf die Digitalisierungsstraße.

Auf Basis des erweiterten Wissens wurde in der zweiten Iteration der Digitalisierungsstraße Citizen Sensing als Spezifizierung der Bürgerbeteiligung in der weiteren Entwicklung fokussiert. Mit den Ergebnissen des Evaluationsworkshops konnten auf Basis des Feedbacks der Experten die Anforderungen und Struktur der Digitalisierungsstraße angepasst werden. Auf Grund der steigenden Komplexität der Umge- 
bung und der beteiligten Akteure wurde für die zweite Iteration „The Four Cycle of Design Science Research“ von Drechsler und Hevner (2016) verwendet. Dieser teilt das Umfeld in eine interne und eine externe Umgebung und erzeugt eine organisatorische und gesellschaftliche Veränderung in der Digitalisierungsstraße. Die beiden Umfelder werden mittels eines vierten Zyklus, dem Change and Impact Cycle, miteinander verbunden. Das interne Umfeld ist der direkte Anwendungskontext des Nutzers, während das externe Umfeld das umfassende sozio-technische System darstellt. Zur Evaluierung der zweiten Iteration wird die Digitalisierungsstraße zunächst als funktionaler Prototyp anhand von sechs Designprinzipien für Plattformen (Giessmann und Legner 2016) umgesetzt und mit Experten in einem zweiten Evaluierungsworkshop getestet. Abb. 1 zeigt die Durchläufe der Zyklen in der zweiten Iteration.

\subsection{Collaboration Engineering}

Um die Effektivität und Effizienz von Aufwendungen und Bemühungen von Individuen in organisatorischen Systemen zu erhöhen, wird Collaboration Engineering eingesetzt (Leimeister 2014). Dabei handelt es sich um einen systematischen Ansatz für die Gestaltung, Entwicklung und Umsetzung von wiederholbaren Kollaborationsprozessen zur Erfüllung von wiederkehrenden und hochwertigen Aufgaben (Leimeister 2014). Zur Erstellung des Designs für einen kollaborativen Prozess müssen verschiedene, sich gegenseitig beeinflussende und hierarchisch voneinander abhängige Themenfelder berücksichtig werden. Bei den im Sechs-Ebenen-Kollaborationsmodell (SeKMo) aufgeführten Themenfeldern handelt es sich um die folgenden: 1. Kollaborationsziele, 2. Gruppenprodukte, 3. Gruppenaktivitäten, 4. Gruppenprozeduren, 5. Kollaborationswerkzeuge und 6. Kollaborationsverhalten (Leimeister 2014). Die Kollaborationsziele beinhalten angestrebte Ergebnisse oder erwünschte Zustände, die durch das gemeinsame Bemühen der Gruppe erreicht werden können. Bei den Gruppenprodukten geht es um materielle oder immaterielle Ergebnisse und Artefakte, die durch Gruppenarbeit erstellt und vorab mit den Kollaborationszielen abgestimmt wurden. Die zu erfüllenden Teilaufgaben zum Erreichen der Gruppenprodukte werden als Gruppenaktivitäten bezeichnet. Alle eingesetzten Kollaborationstechniken und -muster die zur Durchführung der Gruppenaktivitäten beitragen, werden Gruppenprozeduren genannt. Zum Erreichen des Ziels durch die Gruppe werden Kollaborationswerkzeuge wie Systeme oder Artefakte eingesetzt. Jegliche Aktionen und Aussagen der Teilnehmer unter Verwendung der Werkzeuge zur Erreichung des Gruppenziels wird dem Kollaborationsverhalten zugeordnet. Auf Basis des SeKMo wird zur Erstellung des Entwicklungsprozesses für Kollaborationsprozesse der Kollaborations-Prozess-Design-Ansatz (KoPDA) mit dem Fokus auf der Problemanalyse, dem Design und der Transition von Kollaborationsprozessen eingesetzt (Leimeister 2014). KoPDA setzt sich aus fünf iterativ aufbauenden Stufen zusammen, die jeweils untereinander einen direkten Einfluss bewirken. Die erste Stufe umfasst die Analyse der durch die Gruppe durchzuführenden Aufgaben. In der nächsten Stufe findet eine Zerlegung der Aufgaben in Bezug auf die Zusammenarbeit der einzelnen Stakeholder in mehrere Aktivitäten statt. Anschließend werden in der dritten Stufe geeignete thinkLets (gebündelte Moderationstechniken) 


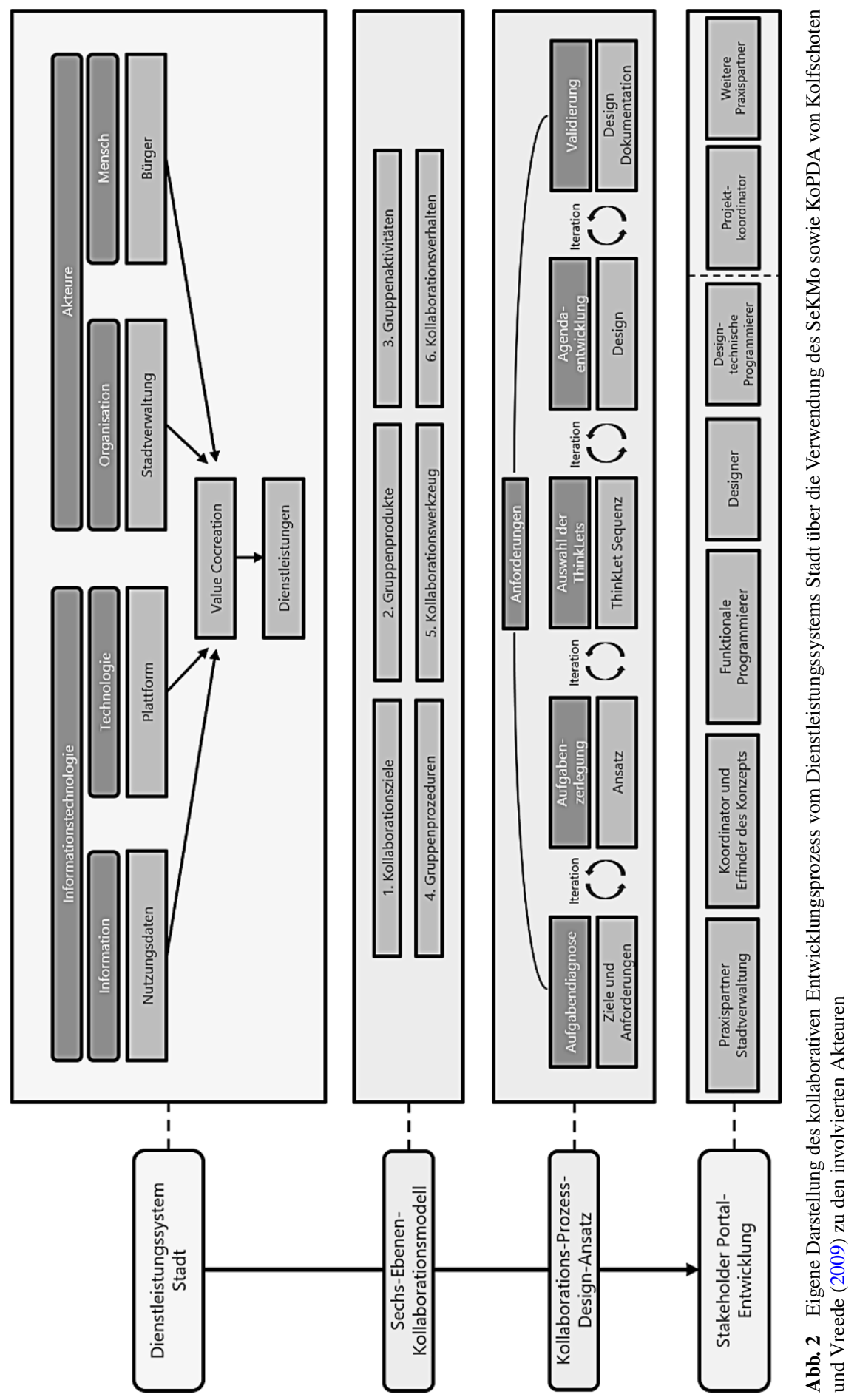


für die einzelnen Aktivitäten auf Basis der Aufteilung angepasst und ausgewählt. Im vierten Schritt wird die Agenda des kollaborativen Prozesses unter Verwendung der thinkLets entwickelt. Zur Überprüfung ob das Design den gewünschten Erfolgt erbracht hat, wird im letzten und fünften Schritt eine Bewertung und Validierung des Designs bzw. des entworfenen Kollaborationsprozesses vorgenommen (Leimeister 2014).

Die kollaborative Erstellung des Portals hat das Ziel die Entwicklung neuer und bedarfsaufgreifender Angebote für die Smart Service Stadt der Zukunft zu fördern, indem Bürgerinnen und Bürger als aktive Mitgestalter von qualitativ hochwertigen Dienstleistungen eingebunden werden. Das Ergebnis soll dabei in einer Stadt mit ca. 200.000 Einwohnern umgesetzt werden. Hierfür entwickeln vier Akteure gemeinsam an der Digitalisierungsstraße. Die Konstellation der Akteure, das Dienstleistungssystem Stadt, das SeKMo sowie der KoPDA nach Kolfschoten und Vreede (2009) werden in Abb. 2 zusammengefasst.

\section{Digitalisierungsstraße: Design und Evaluation}

\subsection{Ergebnisse des Anforderungsworkshops}

Basierend auf dem methodischen Vorgehen werden in diesem Kapitel die dazugehörigen Ergebnisse präsentiert. Zu Beginn des Projekts wurde neben mehreren Literaturanalysen u.a. in den Bereichen „Bürgerbeteiligung“, „Dienstleistungserstellung“ und „Smart City“ auch ein Anforderungsworkshop mit vier Experten aus der Stadtverwaltung und zwei Mitarbeitern einer universitären Einrichtung durchgeführt. Dabei wurden die fünf Kernelemente diskutiert und die Ergebnisse anschließend konsolidiert festgehalten: Ziele, Zielgruppe, Plattform, Meinungsbildung/Abstimmung und Rückmeldung/Austausch zwischen Bürgern und der Stadt für die neue Beteiligungsform. Als (1) Ziele konnten u. a. das Erhalten von guten Ideen der Bürger als Experten auf Basis einer Plattform für Ideen, Projekte und Lösungen identifiziert werden. Des Weiteren sollen die BürgerInnen im Prozess begleitet werden, sodass keine falschen Erwartungen an die Plattform entstehen. Für die (2) Zielgruppe sind ein Internetzugang sowie die Affinität mit einem PC und mit Social-Media-Formaten eine wichtige Voraussetzung. Auf Basis der 90-9-1-Regel (90\% Leser, 9\% Kommentatoren oder Voter und $1 \%$ Erschaffer) konnten unter Berücksichtigung von niedrigen und hohen Erwartungen (High and Low Potentials) die Pioniere (Erschaffer) als Hauptzielgruppe identifiziert werden. Bei der (3) Plattform stellt sich die Frage wie ein Projekt auf der Plattform etabliert werden kann, ob eine Möglichkeit der Nachbesserung der eingereichten Ideen angeboten werden kann und wie ein Projekt definiert ist. Des Weiteren soll eine textuelle Eingabe als Basiseingabe dienen und um ein Foto oder Logo ergänzt werden können. Zudem wäre die Möglichkeit ein Video mit ca. 90 s hochzuladen ein guter Bonus für die Plattform. Als wichtigste Voraussetzung wird die Einreichung eines kommunalen Themas gesehen. Beim Punkt (4) Meinungsbildung/Abstimmung ist die Frage aufgekommen ob der VotingZeitraum auf 6 Termine im Jahr beschränkt werden sollte und ob ein Quorum als Hürde vorhanden sein muss. Für die (5) Rückmeldung/Austausch soll ein Feedback- 
Kanal angeboten werden, über den zu allen Themen Rückmeldung gegeben werden kann - auch außerhalb kommunaler Einflussnahme mit Verweisberatung.

\subsection{Erste Iteration der Digitalisierungsstraße}

Basierend auf den Formen der Bürgerbeteiligung, der Grundlage der Erstellung des Dienstleistungssystems Stadt und den Ergebnissen aus dem Anforderungsworkshop wurde eine erste Iteration der Digitalisierungsstraße erstellt. Dabei werden BürgerInnen systematisch in fünf Schritten von der Idee bis zur ausgearbeiteten Lösungspräsentation begleitet. Im ersten Schritt beschreiben die BürgerInnen in einer Kurzbeschreibung was, für wen und wozu ihre Dienstleistungsidee genutzt werden soll. Der zweite Schritt beinhaltet eine Konkretisierung der Dienstleistungsideen durch eine SWOT-Analyse. Eine SWOT-Analyse dient zur strategischen Planung und analysiert interne Stärken (Strengths) und Schwächen (Weaknesses) sowie externe Chancen (Opportunities) und Risiken (Threats) (Leimeister 2012). Anschließend wird im dritten Schritt eine Handlungsableitung auf Basis der Nutzenstifter und Schmerzensstiller des Value Proposition Canvas durchgeführt. Das Value Proposition Canvas dient zur systematischen Entwicklung des Wertangebots. Es setzt sich zusammen aus dem Kundensegment mit Nutzen, Schmerz und Kunden-Jobs sowie dem Wertangebot mit Nutzen-Stifter, Schmerz-Killer und Produkten \& Services (Leimeister 2015). Im vierten Schritt der Lösungsfindung wird ein Sketch, ein Mockup oder ein Prototyp erstellt, die dazu dienen, die Idee zu visualisieren (Leimeister 2012). Anschließend findet die Lösungspräsentation der Dienstleistungsidee statt, indem aufgezeigt wird für wen welches Problem mit welcher Lösung existiert. Dieser Prozess ist vergleichbar mit dem Schleifen eines Diamanten vom Rohdiamanten (Idee der Bürger) bis zum hochkarätigen Diamanten (ausgearbeitete Dienstleistungslösung). Abb. 3 zeigt die erste Iteration der Digitalisierungsstraße.

\subsection{Erster Evaluierungsworkshop}

Zur Überprüfung der Digitalisierungsstraße wurde ein Offline-Evaluierungs-Workshop mit 12 Experten aus städtischem, universitärem und institutionellem Umfeld durchgeführt. Das Ziel des Workshops war es, die Digitalisierungsstraße durch Experten zu evaluieren, um die Ideen frühzeitig zu validieren, die Anforderungen zu erfassen und schnelles Feedback zur Optimierung der Digitalisierungsstraße mit den fünf Schritten zu erhalten. Dabei wurde pro Schritt eine Karteikarte verwendet, auf denen die 12 Experten eigene Dienstleistungsideen dokumentierten. Für die anschließende Lösungspräsentation der erarbeiteten Dienstleistungsidee standen jedem Beteiligten ein 30-sekündiger Elevator-Pitch zur Verfügung. Im Anschluss an die Präsentation der Dienstleistungslösungen wurden die Experten in drei, gleichgroße, institutionell verschiedene Gruppen eingeteilt, um über die Optimierung der Digitalisierungsstraße zu diskutieren. Die Konsolidierung der Ergebnisse hat ergeben, dass die Digitalisierungsstraße einem logischen Grundmuster mit einer guten Struktur sowie Menüführung folgt und geeignete Methoden zum Einsatz kommen. Jedoch sei die Detailtiefe sowie Dauer zu hoch und die Komplexität zu wissenschaftlich und verpflichtend. Die Aufgabenstellung, Begrifflichkeiten und Wertschätzungen seien 


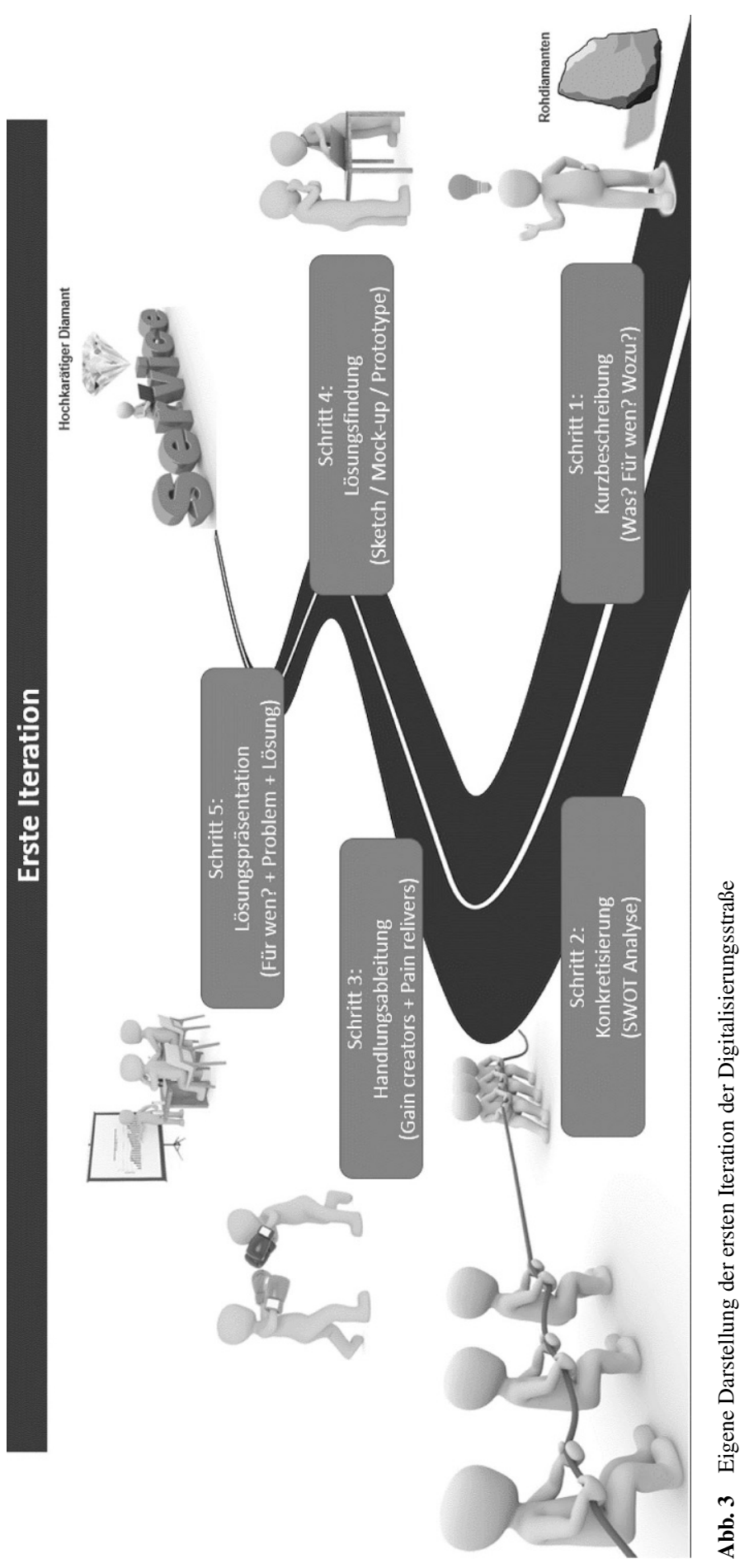


nicht klar formuliert und es existiere kein konkretes Beispiel. Des Weiteren seien zu viele Schritte und Redundanzen in den einzelnen Schritten vorhanden. Hinzu komme ein negativer Ausgangspunkt, fehlende Kollaboration und kein direktes Feedback für jeden sowie eine fehlende Erläuterung zur Verwendung und zum weiteren Vorgehen. Die gerade aufgeführten Beobachtungen und Anforderungen sind in die zweite Entwicklungsiteration der Digitalisierungsstraße eingeflossen.

\subsection{Zweite Iteration der Digitalisierungsstraße}

Basierend auf den konsolidierten Ergebnissen des Evaluationsworkshops und der Erkenntnis aus der bestehenden Literatur, dass Citizen Sensing als neue Form der Bürgerbeteiligung den Bürger zentriert betrachtet, wurde die Digitalisierungsstraße neu gestaltet. Im ersten Schritt, der Kurzbeschreibung der Projekte, erklären die BürgerInnen ihr Projekt anhand mehrerer Schlüsselfragen und lokalisieren es auf einer Karte. Zu den Schlüsselfragen zählen: „Wie heißt das Projekt?“, ,Was ist das Ziel des Projekts?“, „Warum ist das Projekt wichtig?“, „Für wen ist das Projekt gedacht?“ und „Wie soll das Projekt umgesetzt werden?“. Außerdem können die BürgerInnen das Projekt mit Schlüsselwörtern versehen (,Welche Schlüsselwörter sind mit dem Projekt verbunden?“). Im zweiten Schritt, Projekt in Bildern (visuelle Darstellung), präsentieren die BürgerInnen ihr Projekt auf visueller Ebene. Dazu können die BürgerInnen entweder Fotos vom Projekt hochladen oder die vorhandene Zeichenumgebung mit den bereitgestellten Designelementen nutzen. Im dritten und letzten Schritt, Projekt als Video (Elevator-Pitch), fassen die BürgerInnen zusammen, was sie mit ihrem Projekt erreichen möchten, für wen das Projekt gedacht ist und warum das Projekt wichtig ist. Dies kann durch Beschreiben, Sprechen oder Filmen erfolgen und basiert in der Regel auf dem evaluierten Elevator-Pitch-Framework. Die BürgerInnen haben anschießende die Möglichkeit über die einzelnen Projekte abzustimmen. Abb. 4 fasst die drei Schritte der Digitalisierungsstraße zusammen.

\subsection{Technische Umsetzung der Digitalisierungsstraße}

Zur Umsetzung der Digitalisierungsstraße als Portal auf einer Plattform wird die Open-Source-Software Liferay Portal ${ }^{2}$ verwendet. Liferay Portal basiert auf Java und existiert in zwei verschiedenen Versionen - einer kommerziellen Enterprise Edition und einer kostenlosen Community Edition, wobei in unserem Fall Letzteres Verwendung findet. Im Liferay Portal werden sogenannte Portlets eingesetzt, die eine Erstellung von sozialen, dynamischen Systemen ermöglichen und sich aus in sich interaktiv, abgeschlossene Elementen zusammensetzen. Dafür steht ein Entwicklungsframework für neue Anwendungen und Anpassungen sowie bereits vorhandene Standardanwendungen zur Verfügung. Einerseits wurden für die Entwicklung des Portals Standard-Portlets verwendet, wie der Registrierungsprozess bei der Anmeldung. Andererseits mussten Portlets komplett neu erstellt werden, z. B. die webbasierte Zeichenumgebung, oder bestehende Portlets mussten angepasst werden, wie der Upload und die Konvertierung von Videodateien. Abb. 5 zeigt den ersten

\footnotetext{
2 https://www.liferay.com/.
} 


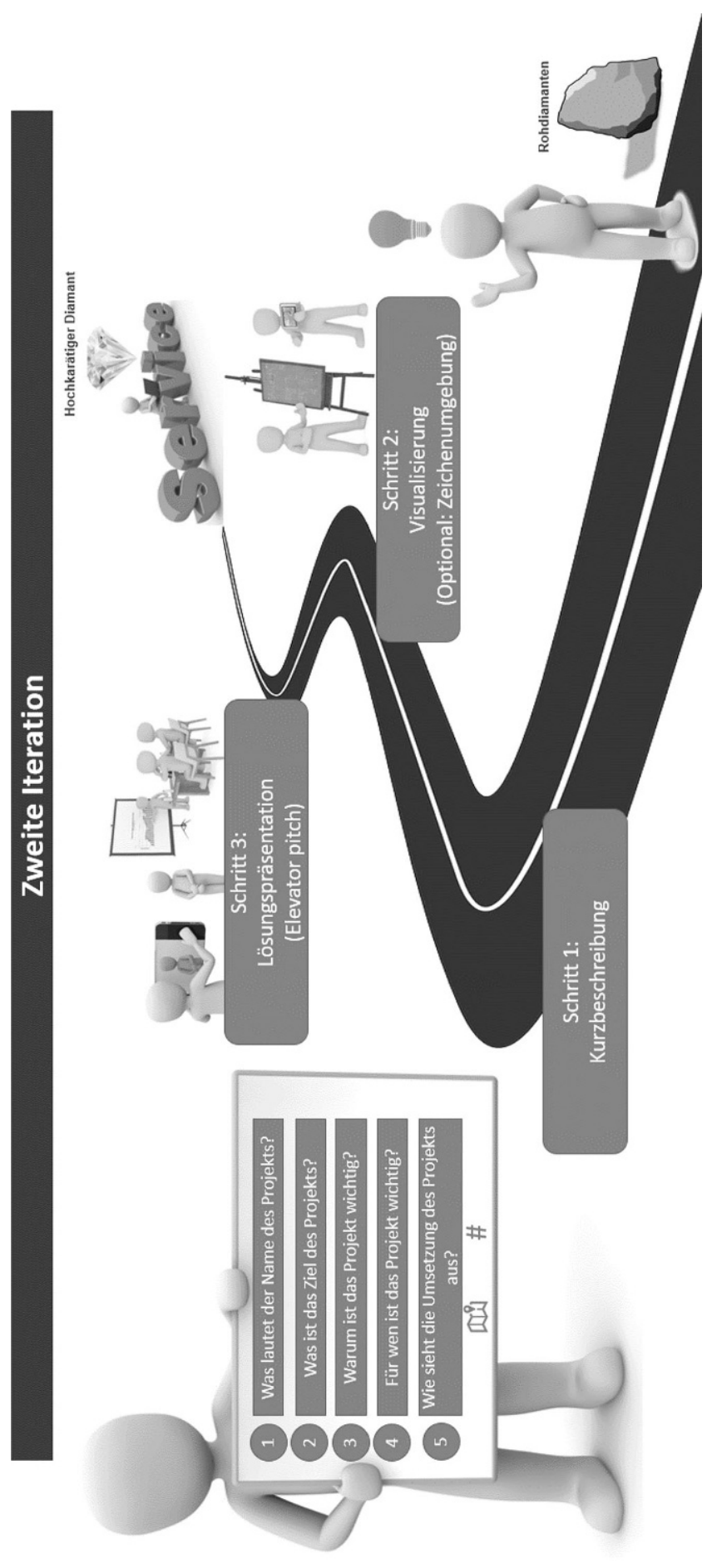

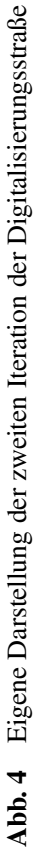




\section{Ein neues Projekt einreichen}

(1) Kurzbeschreibung

Bitte beantworten Sie alle Fragen, um eine genaue Beschreibung Ihres Projekts zu erstellen.
Wie lautet der Name des Projekts?

Was ist das Ziel des Projekts?

Für wen ist das Projekt wichtig?

Warum ist das Projekt wichtig?

Wie sieht die Umsetzung des Projekts aus?

Welche Schlagworte sind mit dem Projekt verbunden?

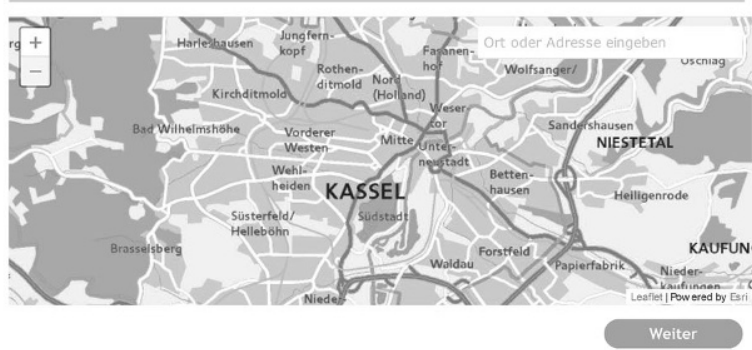

Ober FAQ AGB Impressum Datenschutz
Klicken Sie auf die Karte, um das Projekt zu verorten.

Abb. 5 Ausschnitt aus dem Portal: Der erste Schritt der Digitalisierungsstraße 
Schritt der Digitalisierungsstraße, einen Ausschnitt des Portals aus der auf Liferay basierenden Plattform.

Zur Evaluierung des Prototypens wurde ein zweiter Evaluierungsworkshop mit den gleichen Experten des ersten Workshops durchgeführt. Die Experten durchliefen erneut die Digitalisierungsstraße, diesmal digital und im Rahmen der drei beschriebenen Schritte.

\section{Limitationen und zukünftige Forschungsaspekte}

Zum aktuellen Stand dieses Beitrags ist der Prototyp der Digitalisierungsstraße zur Erstellung von bürgerinitiierten Dienstleistungen noch nicht vollständig fertiggestellt und einige Issues aus der Übersicht der Sprint-Meetings müssen noch eingearbeitet werden. Bevor die Digitalisierungsstraße an die Prozesse der Stadtverwaltung angebunden wird, sind zunächst noch weitere Experimente und Labortests sowie Feldversuche mit potentiellen Nutzern notwendig, um die Digitalisierungsstraße bürgerzentriert und den Erwartungen entsprechend zu gestalten. Des Weiteren wird in einem nächsten Schritt die Digitalisierungsstraße weiter ausgebaut, einerseits um die Weiterentwicklung der Dienstleistungsideen bis zur möglichen Implementierung auf Basis des Service Engineerings (Leimeister 2012) und des Collaboration Engineerings (Leimeister 2014). Andererseits muss ein Anreizsystem für die BürgerInnen geschaffen werden, damit diese die Dienstleistungsideen über die Digitalisierungsstraße generieren und auch weiterentwickeln. Hierfür soll eine auf Blockchain-basierte Kryptowährung als Belohnungssystem auf der Plattform etabliert werden. Damit die nutzerorientierte und soziale Perspektive sowie Struktur der Digitalisierungsstraße frühzeitig überprüft und der Ansatz nutzer-, nutzungs- und nutzenorientiert optimieren werden kann, ist die Durchführung weiterer Evaluation geplant. Eine Evaluation wird über eine quantitative Befragung der Bürger stattfinden, bei der die BürgerInnen über die neue Beteiligungsart und Optimierungsvorschläge für die Digitalisierungsstraße befragt werden. Weitere einzelne und spezifische Evaluationsepisoden stehen noch nicht fest und sind Teil der weiteren Forschung. Die Nutzung der Bürgerressourcen, die Bürgerzufriedenheit und die Sicherung der Servicequalität konnten aus den Ergebnissen der Workshops als abstrahierte Merkmale identifiziert werden, die es bei der Weiterentwicklung zu berücksichtigen gilt.

Die Digitalisierungsstraße soll die BürgerInnen als Experten ihres Alltags bei der Dienstleistungserstellung systematisch unterstützen und einen einfachen Zugang bieten. Allerdings besitzt die Digitalisierungsstraße im aktuellen Zustand auch Einschränkungen, obwohl es sich um ein an sich logisches Konzept handelt. Zu den Limitierungen der Digitalisierungsstraße gehören die widersprüchliche Innovationsbedingung in einer festetablierten Struktur des öffentlichen Sektors sowie die technologiegetriebenen Projektrisiken, die auftreten können. Des Weiteren existiert kein Freiraum für Tests, da im öffentlichen Sektor Ausfälle auf Grund der hohen Verantwortlichkeit vermieden werden. Außerdem scheitern auf Grund von mangelnder Managementqualität, falsch gesetzten Organisations- und Projektzielen sowie mangelnder Kompetenz und mangelnder administrativer Unterstützung ca. $85 \%$ aller ITProjekte (Nam und Pardo 2011). 
In zukünftiger Forschung gilt es die individuellen Beiträge der Bürger hinsichtlich ihrer Auswirkungen auf die Qualität im öffentlichen Dienstleistungssektor und geeigneter Geschäftsmodelle zu analysieren. Ebenfalls interessant wäre die Bewertung der Dienstleistungserbringung und die Bewertung der Messung von Zufriedenheit der Stadtverwaltung als auch von Bürgern. Eine Adaptierbarkeit auf andere Kontexte wäre ebenfalls ein weiterer Forschungsaspekt.

\section{Zusammenfassung}

Der folgende Beitrag zeigt auf, wie mittels gestaltungsorientierter Forschung, in diesem Fall dem DSR-Ansatz nach Drechsler und Hevner (2016) und Ansätzen aus dem Collaboration Engineering (Leimeister 2014), konkret dem Sechs-Ebenen-Kollaborationsmodell (SeKMo) und dem Kollaborations-Prozess-Design Ansatz (KoPDA), ein Bürgerbeteiligungsportal kollaborativ und iterativ entwickelt wird. Dieses Portal erlaubt die systematische Begleitung der Bürger als Experten ihres Alltags bei der Erstellung eigener Dienstleistungen von der Idee bis zur Lösungspräsentation. Dabei haben die BürgerInnen die Möglichkeit, (1) ihren Projektvorschlag zu beschreiben, (2) ihren Projektvorschlag mittels einer webbasierten Zeichenumgebung visuell darzustellen, (3) ihren Projektvorschlag videobasiert zu präsentieren. Durch das innovative, multimediale und bürger-zentrierte Portal wird den Herausforderungen der Stadtverwaltung entgegengewirkt, indem die Kompetenzen der BürgerInnen nicht außen vorgelassen, sondern sinnvoll in den kollaborativen Dienstleistungsprozess eingebunden werden, um mit neuen Dienstleistungen einen vielseitigen, nachhaltigen und lebenswerten Raum zu schaffen.

Zur kollaborativen Entwicklung der Digitalisierungsstraße wurden zunächst in einem Anforderungsworkshop mit allen Beteiligten die angestrebten Ergebnisse und die erwünschten Zustände sowie die Kollaborationsziele festgehalten. Basierend auf der Literatur von Bürgerbeteiligung und Service Engineering sowie den Ergebnissen des Anforderungsworkshops wurde die Digitalisierungsstraße entwickelt. Die anschließende Umsetzung der Digitalisierungsstraße als Portal erfolgte über eine Open-Source-Software.

Dieser Beitrag erweitert dabei die bestehende Literatur der Bürgerbeteiligung und der kollaborativen Dienstleistungsentwicklung um einen neuen Lösungsansatz zur aktiven Bürgerbeteiligung. Dabei wird der Bürger unter der Verwendung von Werkzeugen und Methoden des Service Engineerings und den Ressourcen eines Dienstleistungssystems aktiv in den Prozess der Dienstleistungsentwicklung eingebunden. Außerdem wird aufgezeigt, wie ein Bürgerbeteiligungsportal auf Basis eines bürgernahen, multimedialen Ansatzes zur systematischen Entwicklung von Dienstleistungsideen kollaborativ mit mehreren Akteuren entwickelt werden kann. Zur Erhöhung der Bürgerbeteiligung zeigen wir mit diesem Beitrag den Stadtverwaltungen die Anforderungen und Richtlinien zur Erstellung eines Bürgerbeteiligungsportals auf. Dadurch zeigen wir einerseits wie ein Bürgerbeteiligungsportal an bestehende Beteiligungsprozess der Stadtverwaltung angebunden und durch eine konstante, kollaborative Entwicklung nutzbar gemacht werden kann. Andererseits gleichen wir die Erwartungen sowohl von der Stadtverwaltung als auch von den BürgerInnen 
durch einen bürger-zentrierten, kollaborativen und iterativen Ansatz von Beginn an kontinuierlich ab.

\section{Literatur}

Böhmann T, Leimeister JM, Möslein K (2014) Service systems engineering. Bus Inf Syst Eng 6(2):73-79 Castelnovo W (2016) Co-production makes cities smarter: citizens' participation in smart city initiatives. In: Fugini M, Bracci E, Sicilia M (Hrsg) Co-production in the public sector. Springer, Cham, S $97-117$

Cosgrave E, Arbuthnot K, Tryfonas T (2013) Living labs, innovation districts and information marketplaces: a systems approach for smart cities. Procedia Comput Sci 16:668-677

Drechsler A, Hevner A (2016) A four-cycle model of IS design science research: capturing the dynamic nature of IS artifact design. In: Parsons J, Tuunanen T, Venable JR, Helfert M, Donnellan B, Kenneally J (Hrsg) Breakthroughs and emerging insights from ongoing design science projects 11th International Conference on Design Science Research in Information Systems and Technology (DESRIST), S 1-8 (Research-in-progress papers and poster presentations)

Frecks L (2015) Citizen participation in digital government. 16th Annual International Conference on Digital Government Research—dg.o '15, New York, 27.05.2015-30.05.2015 ACM, New York, S $167-170$

Gabrys J (2014) Programming environments: environmentality and citizen sensing in the smart city. Environ Plan D 32(1):30-48

Giessmann A, Legner C (2016) Designing business models for cloud platforms. Inf Syst J 26(5):551-579

Gregor S, Hevner AR (2013) Positioning and presenting design science research for maximum impact. MIS Q 37(2):337-355

Gutierrez V, Amaxilatis D, Mylonas G, Munoz L (2018) Empowering citizens toward the co-creation of sustainable cities. IEEE Internet Things J 5(2):668-676

Hevner AR (2007) A three cycle view of design science research. Scand J Inf Syst 19(2):4

Kolfschoten GL, de Vreede G-J (2009) A design approach for collaboration processes: a multimethod design science study in collaboration engineering. J Manag Inf Syst 26(1):225-256

Leimeister JM (2012) Dienstleistungsengineering und -management. Springer, Berlin, Heidelberg

Leimeister JM (2014) Collaboration engineering. Springer, Berlin, Heidelberg

Leimeister JM (2015) Einführung in die Wirtschaftsinformatik. Springer, Berlin, Heidelberg

Letaifa SB (2015) How to strategize smart cities: revealing the SMART model. J Bus Res 68(7):1414-1419

Mueller J, Lu H, Chirkin A, Klein B, Schmitt G (2018) Citizen design science: a strategy for crowdcreative urban design. Cities 72(Part A):181-188

Nam T, Pardo TA (2011) Conceptualizing smart city with dimensions of technology, people, and institutions. 12th Annual International Conference on Digital Government Research, S 282-291

Naranjo Zolotov M, Oliveira T, Casteleyn S (2018) E-participation adoption models research in the last 17 years: a weight and meta-analytical review. Comput Hum Behav 81:350-365

Paskaleva K, Cooper I, Concilo G (2018) Co-producing smart city services: Does one size fit all? In: Rodríguez Bolívar M (Hrsg) Smart technologies for smart governments. Public administration and information technology, Bd. 24, S 123-158

United Nations, Department of Economic and Social Affairs, Population Division (2017) World population prospects: the 2017 revision, key findings and advance tables. Working paper no. ESA/P/WP/248

Venable J, Pries-Heje J, Baskerville R (2016) FEDS: a framework for evaluation in design science research. Eur J Inf Syst 25(1):77-89

Viale Pereira G, Cunha MA, Lampoltshammer TJ, Parycek P, Testa MG (2017) Increasing collaboration and participation in smart city governance: a cross-case analysis of smart city initiatives. Inf Technol Dev 23(3):526-553

van Waart P, Mulder I, de Bont C (2016) A participatory approach for envisioning a smart city. Soc Sci Comput Rev 34(6):708-723 